\title{
Dislocation Structures in Low-Angle Grain Boundaries of $\alpha-\mathrm{Al}_{2} \mathrm{O}_{3}$
}

\author{
Eita Tochigi ${ }^{1, *(1)}$, Atsutomo Nakamura ${ }^{2}$ (D), Naoya Shibata ${ }^{1,3}$ and Yuichi Ikuhara $1,3,4$ (iD) \\ 1 Institute of Engineering Innovation, The University of Tokyo, 2-11-16 Yayoi, Bunkyo-ku, Tokyo 113-8656, \\ Japan; shibata@sigma.t.u-tokyo.ac.jp (N.S.); ikuhara@sigma.t.u-tokyo.ac.jp (Y.I.) \\ 2 Department of Materials Physics, Nagoya University, Furo-chou, Chikusa-ku, Nagoya, Aichi 464-8603, \\ Japan; anaka@nagoya-u.jp \\ 3 Nanostructures Research Laboratory, Japan Fine Ceramics Center, 2-4-1, Mutsuno, Atsuta-ku, Nagoya, \\ Aichi 456-8587, Japan \\ 4 Center for Elements Strategy Initiative for Structure Materials, Kyoto University, Yoshidahonmachi, \\ Sakyo-ku, Kyoto 606-8501, Japan \\ * Correspondence: tochigi@sigma.t.u-tokyo.ac.jp; Tel.: +81-3-5841-7689
}

Received: 14 February 2018; Accepted: 7 March 2018; Published: 12 March 2018

\begin{abstract}
Alumina $\left(\alpha-\mathrm{Al}_{2} \mathrm{O}_{3}\right)$ is one of the representative high-temperature structural materials. Dislocations in alumina play an important role in its plastic deformation, and they have attracted much attention for many years. However, little is known about their core atomic structures, with a few exceptions, because of lack of experimental observations at the atomic level. Low-angle grain boundaries are known to consist of an array of dislocations, and they are useful to compose dislocation structures. So far, we have systematically fabricated several types of alumina bicrystals with a low-angle grain boundary and characterized the dislocation structures by transmission electron microscopy (TEM). Here, we review the dislocation structures in $\{11 \overline{2} 0\} /[0001],\{11 \overline{2} 0\} /\langle 1 \overline{1} 00\rangle$, $\{1 \overline{1} 00\} /\langle 11 \overline{2} 0\rangle,(0001) /\langle 1 \overline{1} 00\rangle,\{\overline{1} 104\} /\langle 11 \overline{2} 0\rangle$, and $(0001) /[0001]$ low-angle grain boundaries of alumina. Our observations revealed the core atomic structures of $\boldsymbol{b}=1 / 3\langle 11 \overline{2} 0\rangle$ edge and screw dislocations, $\langle 1 \overline{1} 00\rangle$ edge dislocation, and $1 / 3\langle\overline{1} 101\rangle$ edge and mixed dislocations. Moreover, the stacking faults on $\{11 \overline{2} 0\},\{1 \overline{1} 00\}$, and (0001) planes formed due to the dissociation reaction of the dislocations are discussed, focusing on their atomic structure and formation energy.
\end{abstract}

Keywords: alumina; sapphire; dislocations; low-angle grain boundaries; stacking faults; transmission electron microscopy

\section{Introduction}

A dislocation is one-dimensional lattice defect within a crystal structure. Dislocations strongly influence the mechanical and functional properties of crystalline materials, and thus it is essential to investigate the atomic structures of each dislocation. A dislocation is characterized by its Burgers vector and line direction. The Burgers vector represents the direction and magnitude of lattice distortion due to a dislocation, which is a critical parameter to determining the behavior of a dislocation, such as its slip direction and self-energy. Since the Burgers vector of a perfect dislocation must coincide with a lattice translation vector, the number of possible Burgers vectors is restricted in a crystal structure. In this sense, it should be efficient to characterize dislocation structures systematically in terms of Burgers vector in order to understand the dislocation behavior in a crystal.

A low-angle grain boundary is known to consist of a periodic array of dislocations, and it is useful to design and compose dislocation structures. A low-angle grain boundary is defined as the boundary between two crystal grains with a misorientation typically less than $15^{\circ}[1,2]$. The misorientation of a low-angle grain boundary is accommodated by the presence of dislocations. Low-angle grain 
boundaries are divided into two types: tilt boundaries and twist boundaries, as shown in Figure 1a,b. Basically, a low-angle tilt boundary consists of an array of edge dislocations with the Burgers vector perpendicular to the boundary plane and the line direction parallel to the rotation axis (Figure 1c), whereas a low-angle twist boundary consists of a network of screw dislocations with the Burgers vector on the boundary plane (Figure 1d). The relationship between the interval of perfect dislocations $d$, the Burgers vector $\boldsymbol{b}$, and the misorientation angle of a low-angle grain boundary $\theta$ is given by Frank's equation [1,2]:

$$
d=|\boldsymbol{b}| / \theta .
$$

Note that this equation is valid for both tilt and twist grain boundaries. From these geometrical laws, we can predict the configuration of dislocations in a low-angle grain boundary. In turn, we can compose various dislocation structures by artificially fabricating low-angle grain boundaries.

In this study, we demonstrate the design and characterization of dislocation structures using fabricated low-angle grain boundaries of alumina $\left(\alpha-\mathrm{Al}_{2} \mathrm{O}_{3}\right)$. Alumina has the corundum structure (space group: $R \overline{3} c$ ). The lattice parameters of the hexagonal unit cell are $a=0.476 \mathrm{~nm}$ and $c=1.30 \mathrm{~nm}$ $(c / a=2.73)$. Alumina is one of the representative high-temperature structural materials. Dislocations in alumina play an important role in its plastic deformation processes at elevated temperatures [3-11]. Microstructure analyses of the dislocations using transmission electron microscopy (TEM) were actively performed in the 1970s [4-7]. These studies revealed that the dislocations in deformed alumina crystals are typically dissociated into some partial dislocations with a stacking fault. However, the dislocation core structures, which are critical for the slip behavior, had not been well understood for many years because of a lack of atomic-scale observations. In the 2000s, a high-resolution TEM (HRTEM) study successfully characterized the core structure of $\boldsymbol{b}=1 / 3\langle 11 \overline{2} 0\rangle$ edge dislocation associated with the $(0001)\langle 11 \overline{2} 0\rangle$ basal slip [11]; nevertheless, the core structures of the other types of dislocation were still unidentified. In addition, dislocations in alumina strongly interact with impurity atoms [12-15], and impurity-doped dislocations have a potential to become functional nanowires $[12,16,17]$. To efficiently utilize such functional nanowires, the control of dislocation configuration will be a key technique. Consequently, it is of great interest to investigate the core structures and configurations of dislocations in alumina.

In alumina, the bicrystal method, joining two pieces of single crystal at high temperature, is useful to obtain well-oriented grain boundaries [18-20]. So far, we have systematically fabricated alumina bicrystals with a low-angle grain boundary and characterized the dislocation structures formed in the grain boundaries by TEM [21-32]. The low-angle grain boundaries investigated are tilt boundaries of $\{11 \overline{2} 0\} /[0001]$ [21], $\{11 \overline{2} 0\} /\langle 1 \overline{1} 00\rangle$ [22-26], $\{1 \overline{1} 00\} /\langle 11 \overline{2} 0\rangle$ [27,31], (0001) / [11̄00] [30], and $\{\overline{1} 104\} /\langle 11 \overline{2} 0\rangle[28,32]$, and a twist boundary of $(0001) /[0001]$ [29], as listed in Table 1. In each notation, the indices refer to the grain boundary plane and rotation axis. From the geometrical laws regarding low-angle grain boundaries, it is expected that these five tilt grain boundaries consist of edge dislocations with $\boldsymbol{b}=1 / 3\langle 11 \overline{2} 0\rangle, 1 / 3\langle 11 \overline{2} 0\rangle,\langle 1 \overline{1} 00\rangle$, [0001], and $1 / 3\langle\overline{1} 101\rangle$, respectively. In the last case, the $\{\overline{1} 104\}$ plane and the $1 / 3\langle\overline{1} 101\rangle$ vector are not normal but at an angle of $84.16^{\circ}$ (There is no low-index plane normal to the $1 / 3\langle\overline{1} 101\rangle$ vector.), and the grain boundary should contain not only $1 / 3\langle\overline{1} 101\rangle$ dislocation but also other types of dislocations. In the (0001)/[0001] twist grain boundary, $1 / 3\langle 11 \overline{2} 0\rangle$ screw dislocations are expected to be formed. In this paper, we review the configurations and atomic structures of dislocations in these low-angle grain boundaries.

Table 1. The low-angle grain boundaries investigated in this study.

\begin{tabular}{lccc}
\hline Notation & Type & Misorientation Angle & Burgers Vector Expected \\
\hline$\{11 \overline{2} 0\} /[0001]$ & tilt & $2^{\circ}, 6^{\circ}, 8^{\circ}$ & $1 / 3\langle 11 \overline{2} 0\rangle$ \\
$\{11 \overline{2} 0\} /\langle 1 \overline{1} 00\rangle$ & tilt & $2^{\circ}, 1^{\circ}$ & $1 / 3\langle 11 \overline{2} 0\rangle$ \\
$\{1 \overline{1} 00\} /\langle 11 \overline{2} 0\rangle$ & tilt & $2^{\circ}$ & $\langle 1 \overline{1} 00\rangle$ \\
$(0001) /\langle 1 \overline{1} 00\rangle$ & tilt & $2^{\circ}$ & {$[0001]$} \\
$\{\overline{1} 104\} /\langle 11 \overline{2} 0\rangle$ & tilt & $2^{\circ}$ & $1 / 3\langle\overline{1} 101\rangle$ \\
$(0001) /[0001]$ & twist & $\sim 0^{\circ}$ & $1 / 3\langle 11 \overline{2} 0\rangle$ \\
\hline
\end{tabular}


(a)

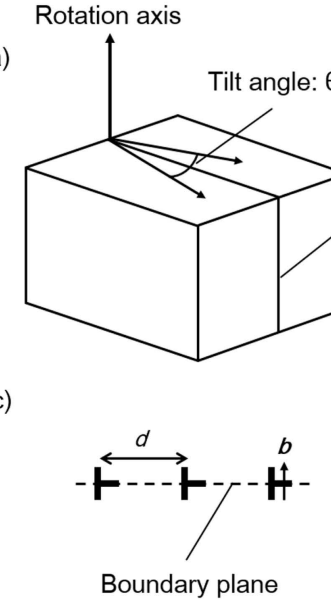

(b)

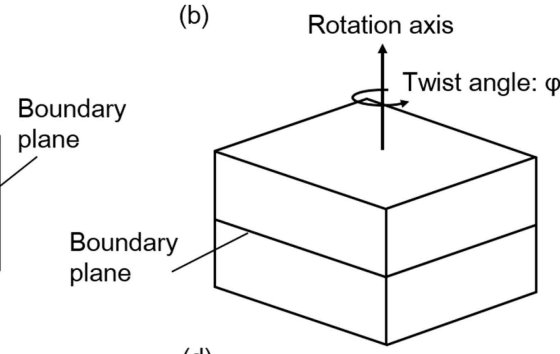

(d)

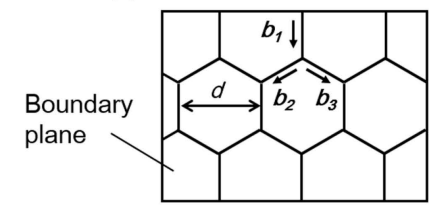

Figure 1. Schematic illustrations of low-angle grain boundaries: (a) tilt boundary; (b) twist boundary. Typical dislocation structures in (c) tilt boundary; (d) twist boundary.

\section{Materials and Methods}

Alumina bicrystals with a low-angle grain boundary were fabricated by joining two pieces of alumina single crystal at $1500{ }^{\circ} \mathrm{C}$ for $10 \mathrm{~h}$ in air. The low-angle grain boundaries examined in this study are listed in Table 1 . The bicrystals were cut to small chips, and then they were thinned by mechanical grinding and Ar ion milling to obtain electron transparency. The samples were observed by TEM (JEOL JEM-2010HC, 200 kV, Tokyo, Japan), high-resolution TEM (HRTEM: Topcon 002BF, 200 kV; JEOL JEM-4010, 400 kV, Tokyo, Japan), and scanning TEM (STEM: JEOL ARM-200F, 200 kV, Tokyo, Japan).

\section{Results and Discussion}

\section{1. $\{11 \overline{2} 0\} /[0001]$ Low-Angle Tilt Grain Boundary}

Figure 2a shows a TEM image of the $\{11 \overline{2} 0\} /[0001] 2^{\circ}$ low-angle tilt grain boundary. Pair contrasts are periodically arrayed with the interval of about $13.2 \mathrm{~nm}$, suggesting that each dislocation is dissociated into two partial dislocations. The Burgers vector of the dislocation pairs should be $\boldsymbol{b}=1 / 3\langle 11 \overline{2} 0\rangle$ in total because the translation vector of $1 / 3\langle 11 \overline{2} 0\rangle$ is perpendicular to the $\{11 \overline{2} 0\}$ grain boundary plane. Substituting $d=13.2 \mathrm{~nm}$ and $|\boldsymbol{b}|=|1 / 3\langle 11 \overline{2} 0\rangle|=0.476 \mathrm{~nm}$ into Equation (1), the misorientation angle $\theta$ is estimated to be $2.1^{\circ}$, which agrees with the designed angle of the present grain boundary.

A HRTEM image of a dislocation pair in the grain boundary is shown in Figure $2 b$. The two dislocation cores corresponding to partial dislocations are observed. These partial dislocations are separated along the $\{11 \overline{2} 0\}$ plane, suggesting that a stacking fault on the $\{11 \overline{2} 0\}$ plane is formed between the partial dislocations. The plane of the stacking fault does not coincide with the slip plane of the perfect dislocation. Accordingly, the partial dislocations were separated by the self-climb mechanisms. This type of dissociation is called the climb dissociation. The large Burgers circuit shows that this dislocation pair has the Burgers vector of $1 / 3\langle 11 \overline{2} 0\rangle$, and the small Burgers circuits show that the upper and the lower partial dislocations have the Burgers vectors of $1 / 3\langle 10 \overline{1} 0\rangle$ and $1 / 3\langle 01 \overline{1} 0\rangle$, respectively. This dissociation reaction is represented as follows:

$$
1 / 3\langle 11 \overline{2} 0\rangle \rightarrow 1 / 3\langle 10 \overline{1} 0\rangle+1 / 3\langle 01 \overline{1} 0\rangle .
$$

The $\{11 \overline{2} 0\} /[0001]$ grain boundaries with the tilt angle of $6^{\circ}$ and $8^{\circ}$ were also found to consist of the same type of partial-dislocation pairs [21]. It is known that the slip dislocation associated with the $(0001)\langle 11 \overline{2} 0\rangle$ basal slip (the so-called basal dislocation) has the Burgers vector of $1 / 3\langle 11 \overline{2} 0\rangle$, and the 
$1 / 3\langle 11 \overline{2} 0\rangle$ basal edge dislocation also dissociates into two partial dislocations following the reaction of Equation $(2)[4,6,10]$. Note that $1 / 3\langle 11 \overline{2} 0\rangle$ edge dislocation in the $\{11 \overline{2} 0\} /[0001]$ tilt grain boundary has the line direction of [0001], whereas the $1 / 3\langle 11 \overline{2} 0\rangle$ basal edge dislocation has the line direction of $[1 \overline{1} 00]$. Therefore, they are not equivalent to each other.

The $\{11 \overline{2} 0\}$ stacking faults generated by the $1 / 3\langle 10 \overline{1} 0\rangle$ or $1 / 3\langle 01 \overline{1} 0\rangle$ partial dislocations are known to be structurally equivalent $[5,11]$; that is, there is only one type for the $\{11 \overline{2} 0\}$ stacking fault. The formation energy of a stacking fault (stacking fault energy) $\gamma_{S F}$ formed between a partial-dislocation pair can be estimated by calculating the repulsive force acting between the partial dislocations based on an elastic theory, which is the so-called Peach-Kohler equation [2]. For a partial-dislocation pair in a low-angle boundary, contributions from other dislocations also need to take into account the force calculations, and a detailed derivation is given elsewhere $[21,33]$. For the present case, the repulsive force $f\left(=\gamma_{S F}\right)$ is represented as follows:

$$
\gamma_{S F}=f=\frac{\mu b_{p}^{2}}{4 \pi(1-v)} \cdot \frac{1}{d} \sum_{n=0}^{\infty}\left(\frac{1}{n+\alpha}-\frac{1}{n+1-\alpha}\right),
$$

where $\mu$ is the shear modulus ( 150 GPa [34]), $v$ is Poisson's ratio ( 0.24 [35]), $b_{p}$ is the magnitude of the Burgers vector of partial dislocations, $|1 / 3\langle 10 \overline{1} 0\rangle|=0.275 \mathrm{~nm}$, and $\alpha$ is $d_{1} / d\left(d_{1}\right.$ : the spacing of a partial dislocation pair, or the width of the stacking fault). Using the averaged distances measured from our experiment, $d=13.2 \mathrm{~nm}$ and $d_{1}=4.6 \mathrm{~nm}$, the stacking fault energy was estimated to be $0.32 \mathrm{Jm}^{-2}$. This value agrees well with an experimental value calculated from an isolated partial-dislocation pair in a deformed crystal, $0.28 \mathrm{Jm}^{-2}$ [11]. In addition, a couple of theoretical studies have been carried out to examine the $\{11 \overline{2} 0\}$ stacking fault $[36,37]$, and one using first-principles calculations within the generalized gradient approximation proposed a similar value of $0.35 \mathrm{Jm}^{-2}$ [37].

From Equations (1) and (3), the relationship between $d_{1}$ and $\theta$ is given. Since the stacking fault energy does not depend on the tilt angle, $d_{1}$ can be obtained as a function of $\theta$. This indicates that the configuration of partial dislocations dissociated by climb in a low-angle boundary can be predicted by the stacking fault energy corresponding to the grain boundary plane in addition to the orientation relationship.

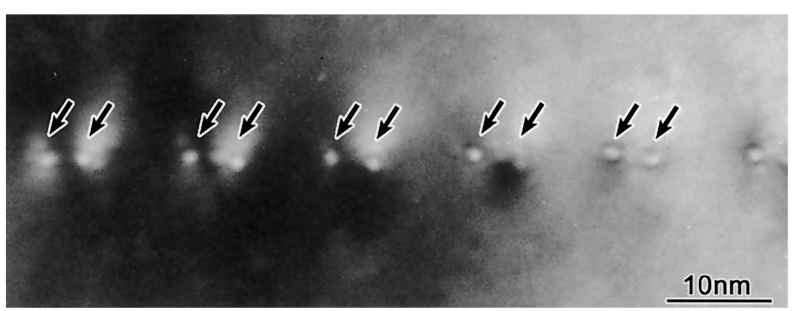

(a)

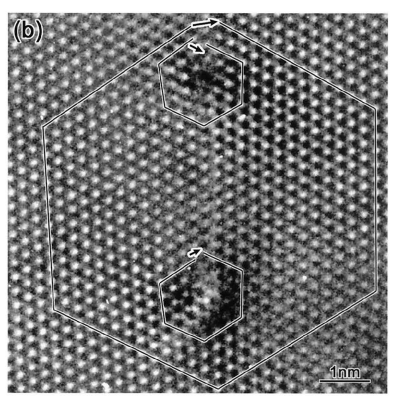

(b)

Figure 2. (a) TEM image of the $\{11 \overline{2} 0\} /[0001] 2^{\circ}$ low-angle tilt grain boundary. Partial-dislocation pairs are formed along the grain boundary. (b) HRTEM image of a partial dislocation pair. The grain boundary is parallel to the vertical direction. The large Burgers circuit shows edge component of $1 / 3\langle 11 \overline{2} 0\rangle$, and the small circuits show edge components of $1 / 3\langle 10 \overline{1} 0\rangle$ and $1 / 3\langle 01 \overline{1} 0\rangle$. The images shown are adapted from [21] and reprinted with the permission of the American Ceramics Society.

\section{2. $\{11 \overline{2} 0\} /\langle 1 \overline{1} 00\rangle$ Low-Angle Tilt Grain Boundary}

Figure 3a shows a TEM image of the $\{11 \overline{2} 0\} /\langle 1 \overline{1} 00\rangle$ low-angle $2^{\circ}$ tilt grain boundary. It is seen that dislocations are periodically arrayed along the grain boundary. The dislocation structure is divided into two groups, pairs of dislocations and groups of odd-numbered dislocations. Figure $3 \mathrm{~b}$ 
shows a dark-field TEM image taken at the same region in Figure 3a using the reflection of $g=\overline{3} 030$, where the $\{11 \overline{2} 0\}$ grain boundary plane is inclined by about $30^{\circ}$ from the observation direction. The dislocations are clearly seen as line contrasts. Figure $3 c$ shows an HRTEM image of a dislocation pair. The dislocation is dissociated into two partial dislocations on the $\{11 \overline{2} 0\}$ plane. The large Burgers circuit shows that the dislocation pair has the Burgers vector of $1 / 3\langle 11 \overline{2} 0\rangle$ in total. The small Burgers circuits show that each partial dislocation has an edge component of $1 / 6\langle 11 \overline{2} 0\rangle$. This component corresponds to the $\{1 \overline{1} 00\}$ projection of the vectors of $1 / 3\langle 10 \overline{1} 0\rangle$ and $1 / 3\langle 01 \overline{1} 0\rangle$. Therefore, it is considered that the observed structure corresponds to the dissociation of the $1 / 3\langle 11 \overline{2} 0\rangle$ edge dislocation into the $1 / 3\langle 10 \overline{1} 0\rangle$ and $1 / 3\langle 01 \overline{1} 0\rangle$ mixed partial dislocations according to the reaction of Equation (2). The $\{11 \overline{2} 0\} /\langle 1 \overline{1} 00\rangle$ low-angle $10^{\circ}$ tilt grain boundary was also investigated and found to consist of the same type of partial-dislocation pairs [25]. The $1 / 3\langle 11 \overline{2} 0\rangle$ edge dislocation formed in the $\{11 \overline{2} 0\} /\langle 1 \overline{1} 00\rangle$ tilt grain boundary has the dislocation line direction of $\langle 1 \overline{1} 00\rangle$, and thus this dislocation structure is considered to be equivalent to that of the $1 / 3\langle 11 \overline{2} 0\rangle$ basal edge dislocation associated with the $(0001)\langle 11 \overline{2} 0\rangle$ basal slip [10].

The distances between dislocations $d$ and $d_{1}$ were measured to be $15 \mathrm{~nm}$ and $3.6 \mathrm{~nm}$. Using Equation (3) (where the coefficient attributes $\frac{\mu b_{p}^{2}(2+v)}{8 \pi(1-v)}$ ), the formation energy of the $\{11 \overline{2} 0\}$ stacking fault was estimated to be $0.30 \mathrm{Jm}^{-2}$ [24]. This value is consistent with that estimated using the $\{11 \overline{2} 0\} /\langle 0001\rangle$ low-angle tilt grain boundary, as discussed in Section 3.1.

For the imaging condition of $g=\overline{3} 030$ in Figure $3 b$, the partial dislocations with $\boldsymbol{b}_{1}=1 / 3\langle 10 \overline{1} 0\rangle$ and with $\boldsymbol{b}_{2}=1 / 3\langle 01 \overline{1} 0\rangle$ have strong and weak contrasts, respectively. As seen in Figure $3 \mathrm{~b}$, the partial-dislocation pairs $\left(\boldsymbol{b}_{\mathbf{1}}-\boldsymbol{b}_{2}\right.$ pairs) are imaged as the strong and weak line contrasts. From the contrast features, the configurations of the group of five and thirteen partials are found to be $\boldsymbol{b}_{1} \boldsymbol{b}_{1} \boldsymbol{b}_{2} \boldsymbol{b}_{\mathbf{1}} \boldsymbol{b}_{\mathbf{1}}$ and $b_{1} b_{1} b_{2} b_{1} b_{2} b_{1} b_{2} b_{1} b_{2} b_{1} b_{2} b_{1} b_{1}$. The edge components of the partial dislocations with $b_{1}$ and $b_{2}$ are both $1 / 6\langle 11 \overline{2} 0\rangle$, whereas their screw components are $1 / 6\langle 1 \overline{1} 00\rangle$ and $1 / 6\langle\overline{1} 100\rangle$, respectively. Therefore, it is found that the 5-partial structure has an edge component of $5 / 6\langle 11 \overline{2} 0\rangle$ and a screw component of $1 / 2\langle 1 \overline{1} 00\rangle$ in total, and the 13-partial structure has an edge component of $13 / 6\langle 11 \overline{2} 0\rangle$ and a screw component of $1 / 2\langle 1 \overline{1} 00\rangle$. Note that both of the structures have the same screw component. This suggests that the odd numbered dislocation structures are generated by the twist component of the grain boundary. The averaged interval of the odd-numbered dislocation structures was about $230 \mathrm{~nm}$. Substituting $d=230 \mathrm{~nm}$ and $|\boldsymbol{b}|=|1 / 2\langle 1 \overline{1} 00\rangle|=0.407 \mathrm{~nm}$ into Equation (1), the twist angle is estimated to be $0.10^{\circ}$. This value is possible considering the accuracy of the bicrystal fabrication processes.

The odd-numbered dislocation structures can be written by the general expression as:

$$
n \times \frac{1}{3}\langle 11 \overline{2} 0\rangle+\langle 10 \overline{1} 0\rangle \rightarrow(n+3) \times \frac{1}{3}\langle 10 \overline{1} 0\rangle+n \times \frac{1}{3}\langle 01 \overline{1} 0\rangle,
$$

where $n$ is an integer. The odd-numbered dislocation structures with the $n$-value of $0-7$ (corresponding to 3 to 17-partial structures) have been found so far $[22,26]$. The characteristic dislocation configuration in the present grain boundary is explained as follows. An additional twist component to the $\{11 \overline{2} 0\} /\langle 1 \overline{1} 00\rangle$ low-angle tilt grain boundary generates the $\langle 10 \overline{1} 0\rangle$ mixed dislocations into the $1 / 3\langle 11 \overline{2} 0\rangle$ edge dislocation array. The $\langle 10 \overline{1} 0\rangle$ dislocation dissociates into three $1 / 3\langle 10 \overline{1} 0\rangle$ partial dislocations with the $\{11 \overline{2} 0\}$ stacking faults, which are equivalent to that formed between the partial-dislocation pair associated with the $1 / 3\langle 11 \overline{2} 0\rangle$ dislocation. As a result, the partial dislocations generated from the $\langle 10 \overline{1} 0\rangle$ and $1 / 3\langle 11 \overline{2} 0\rangle$ dislocations are combined into the odd-numbered partial structures. In addition, there is a variation of the $n$-values for the odd-numbered partial structures. This would be because the competition between the strain energy and the excess energy of stacking faults. The net screw component of the partial structures with smaller $n$-values is more localized than that with larger $n$ values, namely that the strain energy decreases with the $n$-value. In contrast, the area of stacking fault increases with the $n$-value. 

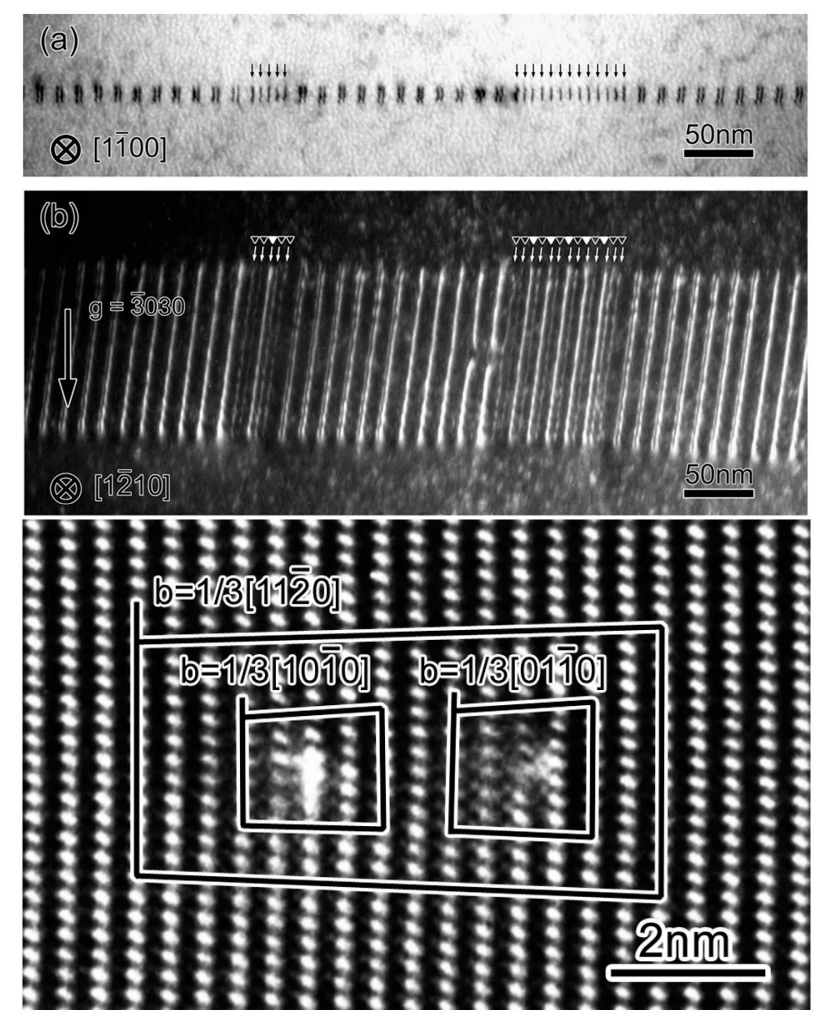

(c)

Figure 3. (a) TEM image of the $\{11 \overline{2} 0\} /\langle 1 \overline{1} 00\rangle 2^{\circ}$ low-angle tilt grain boundary. The grain boundary consists of partial-dislocation pairs and groups of 5 and 13 partial dislocations. (b) dark-field TEM image taken at the same region in (a) using $g=\overline{3} 030$, where the grain boundary plane is inclined by about $30^{\circ}$. Open and filled triangles indicate $1 / 3\langle 10 \overline{1} 0\rangle$ and $1 / 3\langle 01 \overline{1} 0\rangle$ partial dislocations, respectively. (c) HRTEM image of a partial-dislocation pair viewed along the $[1 \overline{1} 00]$ zone axis. From the Burgers circuits, it is found that the dislocation pair has the Burgers vector of $1 / 3\langle 11 \overline{2} 0\rangle$ and the partial dislocations have $1 / 3\langle 10 \overline{1} 0\rangle$ and $1 / 3\langle 01 \overline{1} 0\rangle$. The images $(\mathbf{a})$ and $(\mathbf{b})$ are adapted from [26] and reprinted with the permission of Elsevier B.V. (Amsterdam, Netherlands).

\section{3. $\{1 \overline{1} 00\} /\langle 11 \overline{2} 0\rangle$ Low-Angle Tilt Grain Boundary}

Figure 4a shows a TEM image of the $\{1 \overline{1} 00\} /\langle 11 \overline{2} 0\rangle 2^{\circ}$ tilt grain boundary. Dislocation triplets are arrayed along the grain boundary, suggesting that the dislocations dissociated into three partial dislocations with $\{1 \overline{1} 00\}$ stacking faults. An HRTEM image of a dislocation triplet is shown in Figure $4 \mathrm{~b}$. The three partial dislocations connected with the two stacking faults are clearly observed. The Burgers circuit shows that this dislocation triplet has the Burgers vector of $\langle 1 \overline{1} 00\rangle$ in total. Therefore, this dissociation reaction is written as:

$$
\langle 1 \overline{1} 00\rangle \rightarrow 1 / 3\langle 1 \overline{1} 00\rangle+1 / 3\langle 1 \overline{1} 00\rangle+1 / 3\langle 1 \overline{1} 00\rangle
$$

This dissociation reaction is known to occur for the $\langle 1 \overline{1} 00\rangle$ slip dislocation associated with the $\{11 \overline{2} 0\}\langle 1 \overline{1} 00\rangle$ prism-plane slip [5].

The two stacking faults on the $\{1 \overline{1} 00\}$ plane are formed between three partial dislocations. The stacking sequence of the $\{1 \overline{1} 00\}$ plane is represented as ...ABCABC... Thus, the stacking faults generated by the fault vector of $1 / 3\langle 1 \overline{1} 00\rangle$ have structural variations: ...ABC/B/ABC..., $\ldots \mathrm{ABC} / \mathrm{C} / \mathrm{ABC} \ldots$, and $\ldots \mathrm{ABC} / / \mathrm{BCA} \ldots$, where the position of stacking disorder is indicated by '//'. These stacking faults are called interstitial fault type-I $\left(\mathrm{I}_{1}\right)$, interstitial fault type-II $\left(\mathrm{I}_{2}\right)$, and vacancy fault $(\mathrm{V})$, respectively $[8,27]$. Due to the geometric constraint, the combination of the stacking faults 
formed between the $1 / 3\langle 1 \overline{1} 00\rangle$ triplet is known to be either of $I_{1}-V$ or $I_{2}-V[8,27]$. To identify the structure of the stacking faults formed between the partial dislocations, we observed the stacking faults by atomic-resolution STEM.

Figure $4 \mathrm{c}, \mathrm{d}$ show annular bright-field (ABF) STEM images of the stacking faults, corresponding to the left and the right ones in Figure $4 \mathrm{~b}$, respectively. ABF STEM is capable of visualizing atomic columns and even light elements [38,39]. In these images, strong dark contrasts correspond to aluminum columns and weak dark contrasts to oxygen columns, as shown in the atomic structure model in the figure. The stacking sequences of the stacking faults can be directly interpreted as ... $\mathrm{ABC} / \mathrm{C} / \mathrm{ABC} \ldots\left(\mathrm{I}_{2}\right)$ for the left stacking fault and ... ABC//BCAB...: (V) for the right stacking fault.

Their stacking fault energies can be estimated by the similar way as discussed in Section 3.1. For the present case, the equations become the following forms:

$$
\begin{aligned}
& \gamma_{I 2}=\frac{\mu b_{p}^{2}}{2 \pi(1-v)} \cdot \frac{1}{d} \sum_{n=0}^{\infty}\left(\frac{1}{n+\alpha_{1}}+\frac{1}{n+\alpha_{1}+\alpha_{2}}-\frac{1}{n+1-\alpha_{1}}-\frac{1}{n+1-\alpha_{1}-\alpha_{2}}\right), \\
& \gamma_{V}=\frac{\mu b_{p}^{2}}{2 \pi(1-v)} \cdot \frac{1}{d} \sum_{n=0}^{\infty}\left(\frac{1}{n+\alpha_{2}}+\frac{1}{n+\alpha_{1}+\alpha_{2}}-\frac{1}{n+1-\alpha_{2}}-\frac{1}{n+1-\alpha_{1}-\alpha_{2}}\right),
\end{aligned}
$$

where $\alpha_{1}$ is $d_{1} / d\left(d_{1}\right.$ : the width of staking fault $\left.I_{2}\right)$ and $\alpha_{2}$ is $d_{2} / d\left(d_{2}\right.$ : the width of staking fault $\left.V\right)$. The experimental distances of $d, d_{1}$ and $d_{2}$ were measured to be $22 \mathrm{~nm}, 4.7-5.1 \mathrm{~nm}$, and $5.5-5.9 \mathrm{~nm}$, respectively. Using these values, the stacking fault energies were estimated to be $\mathrm{I}_{2}: \gamma=0.41-0.46 \mathrm{Jm}^{-2}$ and $V: \gamma=0.33-0.37 \mathrm{Jm}^{-2}$. Theoretical calculations suggested that the stacking fault energies of $\mathrm{I}_{1}, \mathrm{I}_{2}$, and $V$ are $0.62-0.63 \mathrm{Jm}^{-2}, 0.46 \mathrm{Jm}^{-2}$, and $0.41 \mathrm{Jm}^{-2}$, respectively $[27,37]$. These results agree well with the experimental results.

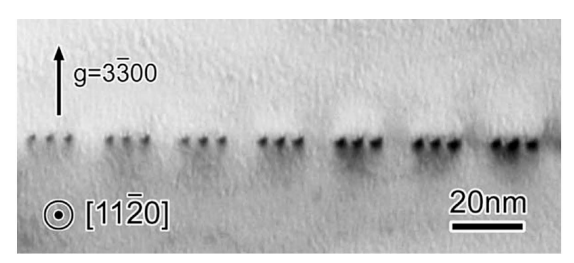

(a)

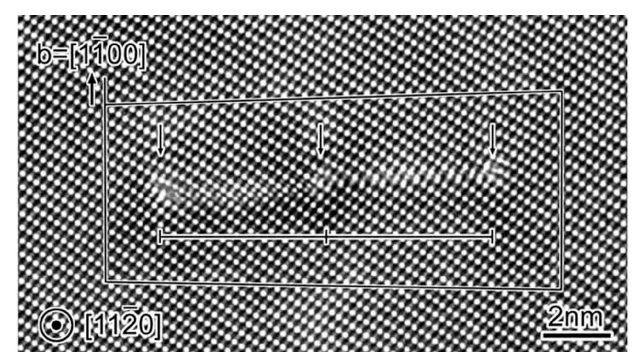

(b)

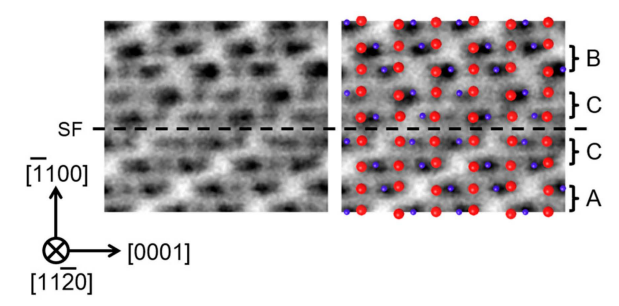

(c)

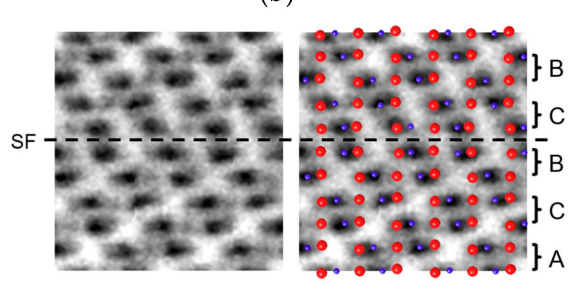

(d)

Figure 4. (a) TEM image of the $\{1 \overline{1} 00\} /\langle 11 \overline{2} 0\rangle 2^{\circ}$ low-angle tilt grain boundary. The grain boundary consists of dislocation triplets. (b) HRTEM image showing one of the dislocation triplets. The Burgers circuit indicates the Burgers vector of $\langle 1 \overline{1} 00\rangle$, suggesting that the $\langle 1 \overline{1} 00\rangle$ dislocation is dissociated into $1 / 3\langle 1 \overline{1} 00\rangle$ partial dislocations with two stacking faults in between. (c) ABF STEM image of the left stacking fault in (b). The atomic structure model overlapped with the image is shown at the right panel. The stacking sequence is ... $\mathrm{ABC} / \mathrm{C} / \mathrm{AB} \ldots: \mathrm{I}_{2}$. (d) The right stacking fault in (b). The stacking sequence is ... ABC//BCAB...: V. The images $(\mathbf{a}, \mathbf{b})$ are adapted from [27] and reprinted with the permission of Elsevier B.V. The images $(\mathbf{c}, \mathbf{d})$ are adapted from [31], a proceedings paper published by AIP Publishing LLC (Melville, New York, NY, US). 


\section{4. $(0001) /\langle 1 \overline{1} 00\rangle$ Low-Angle Tilt Grain Boundary [30]}

Figure 5a shows an HRTEM image of the $(0001) /\langle 1 \overline{1} 00\rangle 2^{\circ}$ low-angle tilt grain boundary. Five dislocation structures are observed and each dislocation structure appears to consist of two partial dislocations with a stacking fault on the (0001) plane. From the Burgers circuits, the edge component of these dislocations is either of $1 / 6[\overline{11} 22], 1 / 6[11 \overline{2} 2]$, or $1 / 3[0001]$. These components do not correspond to a translation vector, and thus these dislocations should be a mixed dislocation with a screw component along the $[1 \overline{1} 00]$ direction. The screw components can be uniquely determined so as to match a possible translation vector as follows:

$$
\begin{aligned}
& \text { (edge) (screw) (total) } \\
& 1 / 6[\overline{11} 22]+1 / 6[1 \overline{1} 00]=1 / 3[0 \overline{1} 11] \text {, } \\
& 1 / 6[11 \overline{2} 2]+1 / 6[1 \overline{1} 00]=1 / 3[10 \overline{1} 1] \text {, } \\
& 1 / 3[0001]+1 / 3[\overline{1} 100]=1 / 3[\overline{1} 101] \text {. }
\end{aligned}
$$

The sum of these three vectors is [0001], and their screw components are cancelled out in total. Since the [0001] vector is the translation vector perpendicular to the (0001) boundary plane, it can be said that the tilt component of the grain boundary is effectively accommodated by groups of these three equivalent dislocations of $1 / 3[0 \overline{1} 11], 1 / 3[10 \overline{1} 1]$, and $1 / 3[\overline{1} 101]$. This characteristic dislocation configuration is reasonable in terms of dislocation self-energy, which is proportional to the square of Burgers vector. The magnitude of the [0001] vector is $1.30 \mathrm{~nm}$ and that of $1 / 3\langle\overline{1} 101\rangle$ vector is $0.513 \mathrm{~nm}$, leading the relationship of $\left|\boldsymbol{b}_{[0001]}\right|^{2}>3 \times\left|\boldsymbol{b}_{1 / 3<-1101>}\right|^{2}$. Therefore, the triplet of $1 / 3\langle\overline{1} 101\rangle$ dislocation is considered to be energetically favorable than the single [0001] dislocation.

Figure $5 \mathrm{~b}$ shows an ABF STEM image of the 1/3[1101] dislocation. The zigzag contrasts along the [0001] direction correspond to the configurations of oxygen and aluminum columns as illustrated in the atomic structure at the right. It is clearly seen that the dislocation is dissociated into two partial dislocations with the (0001) stacking fault. The Burgers circuits drawn around the partial dislocations indicate that the left and right ones have an edge component of $1 / 18[11 \overline{2} 3]$ and $1 / 18[1123]$, respectively. Their screw components, which are necessary to determine the dissociation reaction, are not given by the STEM observations. To identify the Burgers vector of the partial dislocations, instead, we analyzed the fault vector of the stacking fault.

An ABF STEM image of the (0001) stacking fault formed between the partial dislocations is shown in Figure $5 \mathrm{c}$. The position of the stacking fault is indicated by the dashed line. As shown in the figure, the stacking sequence along the [0001] direction on the $\{1 \overline{1} 00\}$ projection is $\ldots 12 \mathrm{~A}$ $32 \mathrm{~B} 12 \mathrm{C} 3 / / 1 \mathrm{~A} 2$ 1B $31 \mathrm{C} \ldots$, where the single numbers refer to oxygen layers and the numbers with a letter to aluminum layers. Theoretical calculations revealed that there is the displacement of $1 / 6[\overline{1} 100]$ or $1 / 6[1 \overline{1} 00]$ across the (0001) stacking fault shown in Figure 5c [30], namely that the partial dislocations have a screw component of $1 / 6[\overline{1} 100]$ or $1 / 6[1 \overline{100}]$. Here, the screw components of the partial dislocations are uniquely determined to be $1 / 6[1100]$ because the partial-dislocation pair has the screw component of $1 / 3[1101]$ in total (see Equation (10)). As a result, the Burgers vectors for the two partial dislocations are identified to be $1 / 18[11 \overline{2} 3]+1 / 6[\overline{1} 100]=1 / 18[\overline{2} 4 \overline{2} 3]$ and $1 / 18[\overline{11} 23]+1 / 6[\overline{1} 100]=1 / 18[\overline{4} 223]$. Consequently, the dissociation reaction of the $1 / 3\langle\overline{1} 101\rangle$ mixed dislocations is represented by the following equation:

$$
1 / 3\langle\overline{1} 101\rangle \rightarrow 1 / 18\langle\overline{4} 223\rangle+1 / 18\langle\overline{2} 4 \overline{2} 3\rangle .
$$

The $1 / 3\langle\overline{1} 101\rangle$ dislocation is known to be associated with the $\{1 \overline{1} 02\}\langle\overline{1} 101\rangle$ pyramidal slip (The $\{10 \overline{1} 1\}$ and $\{2 \overline{11} 3\}$ planes are also possible.) [7]. The dissociation reaction of the $1 / 3\langle\overline{1} 101\rangle$ slip dislocation has not been reported.

The formation energy of the (0001) stacking fault can be calculated by a theoretical equation similar to Equation (3). In the present case, however, it should be too complicated to derive such 
theoretical equations because the grain boundary consists of six kinds of partial dislocations. Instead, the (0001) stacking fault energy was numerically calculated using the actual dislocation configurations identified from our experimental observations, and the calculated value was $0.58 \mathrm{Jm}^{-2}$ [30]. In addition, first-principles calculations suggested the stacking fault energy to be $0.72 \mathrm{Jm}^{-2}$ [30].

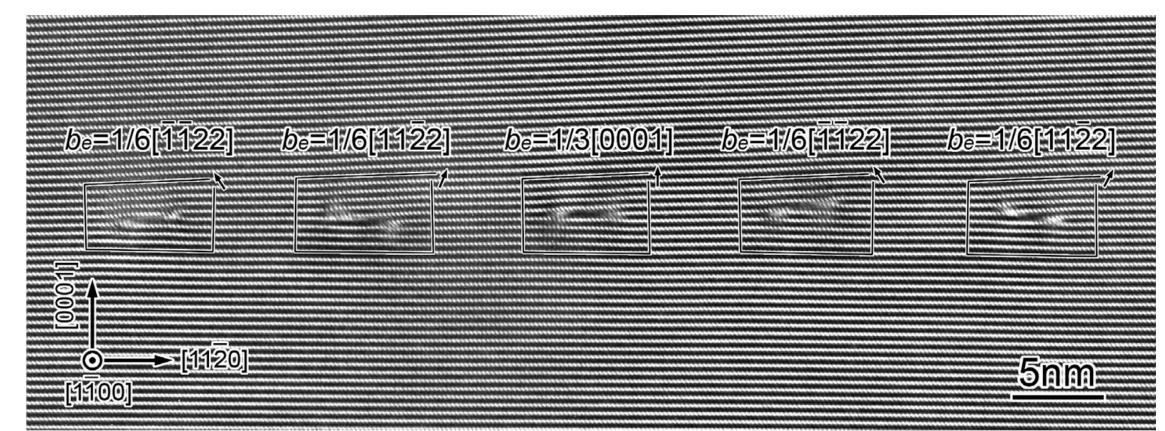

(a)

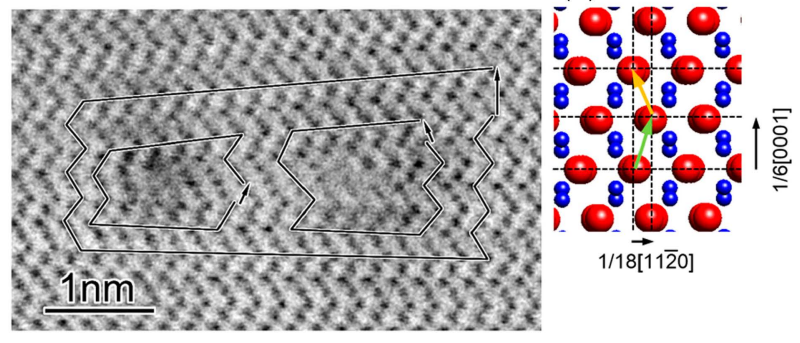

(b)

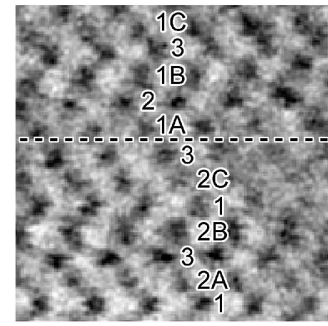

(c)

Figure 5. (a) HRTEM image of the $(0001) /\langle 1 \overline{1} 00\rangle 2^{\circ}$ low-angle tilt grain boundary. The grain boundary consists of dislocations having an edge component of $1 / 6[\overline{11} 22], 1 / 6[11 \overline{2} 2]$, or 1/3[0001]. Each dislocation is dissociated into two partial dislocations with a stacking fault on the (0001) plane; (b) ABF STEM image of the dislocation having an edge component of 1/3[0001]. The Burgers circuits drawn around the partial dislocations indicate edge components of $1 / 18[11 \overline{2} 3]$ and $1 / 18[\overline{11} 23]$, as seen in the atomic structure on the right-hand side. (c) enlarged image of the (0001) stacking fault formed between the partial dislocations. The stacking sequence is represented as ...12A $32 \mathrm{~B} 12 \mathrm{C} 3 / / 1 \mathrm{~A}$ $21 B 31 C \ldots$ in the $\{1 \overline{1} 00\}$ projection. The images $(\mathbf{a}, \mathbf{b})$ are adapted from [30] and reprinted with the permission of Elsevier B.V.

\section{5. $\{\overline{1} 104\} /\langle 11 \overline{2} 0\rangle$ Low-Angle Tilt Grain Boundary}

Figure 6a shows a dark-field TEM image of the $\{\overline{1} 104\} /\langle 11 \overline{2} 0\rangle$ low-angle $2^{\circ}$ tilt grain boundary taken using $g=1 \overline{1} 0 \overline{4}$. The grain boundary appears to be wavy, and relatively broad contrasts and pair contrasts are observed, suggesting that the grain boundary consists of multiple kinds of dislocation structures.

Figure $6 \mathrm{~b}$ shows an HRTEM image of the grain boundary. A single dislocation and two dislocation pairs are observed, as indicated by the arrows. Figure $6 \mathrm{c}$ shows an enlarged image of one of the single dislocations. The single dislocation has an edge component of $1 / 2[\overline{1} 100]$. Since this component does not correspond to a translation vector, the single dislocation should have a screw component along the $[11 \overline{2} 0]$ direction. The screw component is considered to be $1 / 6[11 \overline{2} 0]$ (or 1/6[1120]), which makes the smallest translation vector of $1 / 3[\overline{1} 2 \overline{1} 0]$ (or $1 / 3[\overline{2} 110]$ ). Therefore, the single dislocation should be the $1 / 3[1 \overline{2} 10]$ mixed dislocation. An HRTEM image of a dislocation pair dislocation is shown in Figure $6 \mathrm{~d}$. This dislocation structure consists of two partial dislocations with a stacking fault on the (0001) plane. The Burgers circuits show that the total edge component is $1 / 3[\overline{1} 101]$, and each partial dislocation has an edge component of $1 / 6[\overline{1} 101]$. Since $1 / 3[\overline{1} 101]$ vector corresponds to a translation vector, this dislocation structure is considered to be the $1 / 3[\overline{1} 101]$ edge dislocation. 
It was found that the $\{\overline{1} 104\} /\langle 11 \overline{2} 0\rangle$ low-angle tilt grain boundary consists of not only one kind of dislocation structure. This is because there is no translation vector perpendicular to the $\{\overline{1} 104\}$ grain boundary plane. The $1 / 3[1101]$ vector corresponding to the dislocation pair is at an angle of $84.16^{\circ}$ to the $\{\overline{1} 104\}$ grain boundary plane. This vector introduces a component parallel to the grain boundary of $|1 / 3[\overline{1} 101]| \times \cos 84.16^{\circ}=0.052 \mathrm{~nm}$ (along the $[2 \overline{2} 01]$ direction) in addition to the component normal to the grain boundary. This additional component should be cancelled out in total over the grain boundary by another vector. The $1 / 2[\overline{1} 100]$ vector corresponding to the edge component of the $1 / 3[\overline{1} 2 \overline{1} 0]$ dislocation is at an angle of $141.76^{\circ}$ to the $\{\overline{1} 104\}$ plane. This vector has a component along the $[2 \overline{2} 01]$ direction of $|1 / 2[\overline{1} 100]| \times \cos 141.76^{\circ}=-0.32 \mathrm{~nm}$, which can compensate the additional components due to the $1 / 3[\overline{1} 101]$ vector. Therefore, it is considered that the $1 / 3[\overline{1} 101]$ dislocation pairs and the $1 / 3[\overline{1} 2 \overline{1} 0]$ dislocations are mixed in the grain boundary so as to cancel out a component along the [2201] direction. The ideal ratio of the numers of the $1 / 3[\overline{1} 101]$ dislocation pairs to the $1 / 3[\overline{1} 2 \overline{1} 0]$ dislocations is estimated to be $0.32 / 0.052=\sim 6.2$.

Here, we further discuss the structure of the $1 / 3[1101]$ dislocation pair. Theoretical calculations revealed that the (0001) stacking fault formed between the partial dislocations has a displacement of $1 / 18[1120]$ and its structure is identical to the (0001) stacking fault shown in Figure 5c [32]. The dislocation pair has no screw component in total, and thus the Burgers vectors of the partial dislocations are $1 / 6[\overline{1} 101]+1 / 18[\overline{11} 20]=1 / 18[\overline{4} 223]$ and $1 / 6[\overline{1} 101]-1 / 18[\overline{11} 20]=1 / 18[\overline{2} 4 \overline{2} 3]$. Consequently, it is found that the dissociation reaction of the $1 / 3[1101]$ edge dislocation also corresponds to Equation (11).

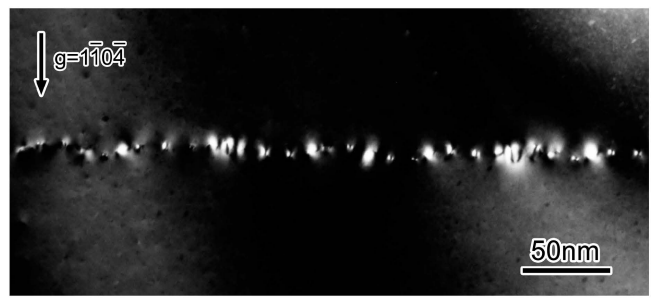

(a)

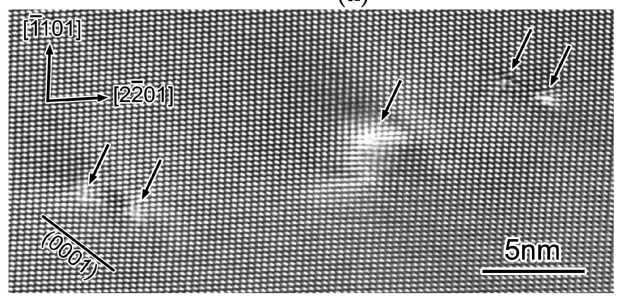

(b)

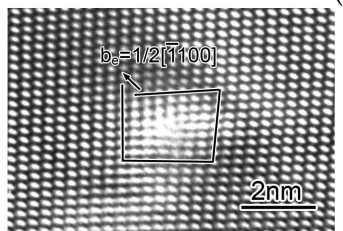

(c)

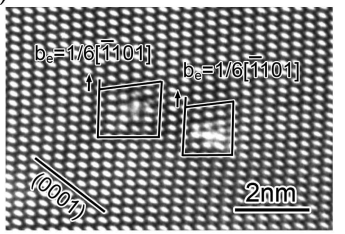

(d)

Figure 6. (a) dark-field TEM image of the $\{\overline{1} 104\} /\langle 11 \overline{2} 0\rangle$ low-angle $2^{\circ}$ tilt grain boundary taken using $g=1 \overline{1} 0 \overline{4}$. In the grain boundary, relatively broad contrasts and pair contrasts are seen. (b) HRTEM image of the grain boundary. A single dislocation and two dislocation pairs are observed. The arrows indicate the dislocation core positions. (c) enlarged image of the single dislocation. This dislocation has an edge component of $1 / 2[\overline{1} 100]$. (d) enlarged image of the dislocation pair. Dislocation is dissociated into partial dislocations with a stacking fault on the (0001) plane. The partial dislocations have an edge component of 1/6[1101]. The images shown are adapted from [28] and reprinted with the permission of Springer Nature (Berlin, Germany). 


\section{6. (0001)/[0001] Low-Angle Twist Grain Boundary}

A (0001)/ [0001] low-angle twist grain boundary was fabricated by joining two pieces of (0001) alumina substrate. The twist angle around the [0001] axis was expected to be as much as the cutting accuracy of the substrate sides $\left(<\sim 0.1^{\circ}\right)$. Two TEM samples were prepared: ones for plan-view and for edge-on observations.

Figure 7a shows a plan-view TEM image of the (0001)/[0001] low-angle twist grain boundary. The observation direction is along the [0001] zone axis. A hexagonal dislocation network is formed on the grain boundary. Since these dislocations are parallel to either of the three equivalent directions of $\langle 11 \overline{2} 0\rangle$, they should correspond to the $1 / 3\langle 11 \overline{2} 0\rangle$ screw dislocations. The interval of the equivalent dislocations is about $60 \mathrm{~nm}$. Using Equation (1), the twist angle of this grain boundary is estimated to be $0.45^{\circ}$.

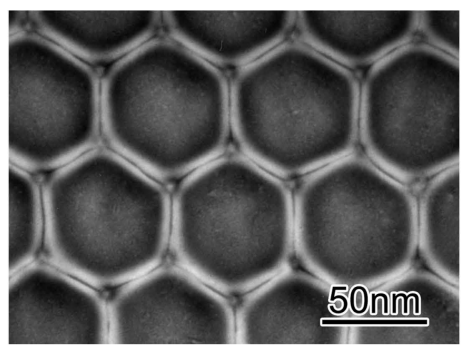

(a)

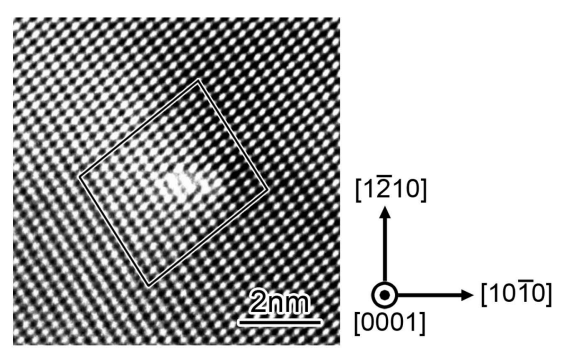

(b)

Figure 7. (a) plan-view TEM image of the (0001) / [0001] low-angle twist grain boundary. A hexagonal

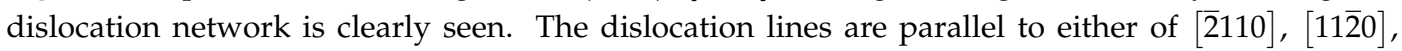
and $[1 \overline{2} 10]$ directions, indicating that they are $1 / 3\langle 11 \overline{2} 0\rangle$ screw dislocations. (b) HRTEM image showing the end-on view of the $1 / 3\langle 11 \overline{2} 0\rangle$ screw dislocation. The screw dislocation is not dissociated. The images shown are adapted from [29] and reprinted with the permission of Elsevier B.V.

An HRTEM image of a $1 / 3\langle 11 \overline{2} 0\rangle$ screw dislocation in the grain boundary viewed end-on is shown in Figure $7 \mathrm{~b}$. The image contrasts are slightly disordered over a few atomic columns, corresponding to the dislocation core region. The circuit drawn around the dislocation core is closed, indicating that this dislocation is a pure screw dislocation. From the image contrasts, the dislocation core is localized within $1 \mathrm{~nm}$ or less, and thus the $1 / 3\langle 11 \overline{2} 0\rangle$ screw dislocation is not dissociated, in contrast to the $1 / 3\langle 11 \overline{2} 0\rangle$ edge dislocation, as discussed in Section 3.2. The $1 / 3\langle 11 \overline{2} 0\rangle$ screw dislocation in the present grain boundary corresponds to the $1 / 3\langle 11 \overline{2} 0\rangle$ basal screw dislocation associated with the $(0001)\langle 11 \overline{2} 0\rangle$ basal slip. The basal screw dislocation is likely to have the perfect type core structure, although its core structure has not been reported. If this is the case, it is considered that the basal screw dislocation slips easily in comparison with the basal edge dislocation. This is because the $\{11 \overline{2} 0\}$ stacking fault generated by the dissociation of the basal edge dislocation is not on the (0001) slip plane and does not move without atomic diffusion.

\section{Findings and Future Subjects}

Our investigations found the dissociation reactions and the core structures of $1 / 3\langle 11 \overline{2} 0\rangle$ (edge, screw), $\langle 1 \overline{1} 00\rangle$ (edge) and $1 / 3\langle\overline{1} 101\rangle$ (edge, mixed) dislocations. The partial dislocations generated by the dissociation reactions of Equations (2), (5), and (11) have the Burgers vector of $1 / 3\langle 10 \overline{1} 0\rangle$ or $1 / 18\langle\overline{4} 223\rangle$. The formation of these two partial dislocations can be understood in terms of the stability of stacking faults. The stacking faults on the $\{11 \overline{2} 0\}$ or $\{1 \overline{1} 00\}$ planes formed by the $1 / 3\langle 10 \overline{1} 0\rangle$ partial dislocation are stable (low energy) (formation energy: 0.3-0.5 Jm${ }^{-2}$ ) [11,21,24,27,37], whereas ones on the (0001) plane were calculated to be unstable $\left(>1 \mathrm{Jm}^{-2}\right)[36,40]$. The stacking fault on the (0001) plane formed by the $1 / 18\langle\overline{4} 223\rangle$ partial dislocation is relatively stable $\left(0.6 \mathrm{Jm}^{-2}\right)[30,32]$. The point is that the stability of stacking faults determines the dissociation reaction of dislocations. To examine whether 
another dissociation reaction is possible, it would be helpful to calculate the relationship between the formation energies of stacking faults and fault vectors for every lattice planes, i.e., generalized stacking fault energies (also known as $\gamma$-surfaces) [41].

Screw dislocations except for the $1 / 3\langle 11 \overline{2} 0\rangle$ dislocation have not been investigated yet. Since the translation vectors of $\langle 1 \overline{1} 00\rangle,[0001], 1 / 3\langle\overline{1} 101\rangle$, and $1 / 3\langle 2 \overline{2} 01\rangle$ are on the $\{11 \overline{2} 0\}$ plane, screw dislocations with either of these vectors are expected to be formed in the $\{11 \overline{2} 0\} /\langle 11 \overline{2} 0\rangle$ low-angle twist grain boundary. Structural analysis of this type of grain boundary will give us further knowledge on screw dislocations in alumina.

\section{Conclusions}

Alumina bicrystals with a low-angle grain boundary were systematically fabricated and the grain boundaries were observed by TEM. The dislocation structures formed in the low-angle grain boundaries are summarized below:

(1) $\{11 \overline{2} 0\} /[0001]$ tilt grain boundary

The $1 / 3\langle 11 \overline{2} 0\rangle$ perfect edge dislocations dissociate into the $1 / 3\langle 10 \overline{0} 0\rangle$ and $1 / 3\langle 01 \overline{1} 0\rangle$ partial dislocations with the $\{11 \overline{2} 0\}$ stacking fault. The stacking fault energy was estimated to be $0.32 \mathrm{Jm}^{-2}$.

(2) $\{11 \overline{2} 0\} /\langle 1 \overline{1} 00\rangle$ tilt grain boundary

The $1 / 3\langle 11 \overline{2} 0\rangle$ perfect edge dislocations dissociate into the $1 / 3\langle 10 \overline{1} 0\rangle$ and $1 / 3\langle 01 \overline{1} 0\rangle$ partial dislocations with the $\{11 \overline{2} 0\}$ stacking fault. The stacking fault energy was estimated to be $0.35 \mathrm{Jm}^{-2}$. It was also found that an additional screw component in the grain boundary forms the odd-numbered partial dislocation structures represented by $(n+3) \times 1 / 3\langle 10 \overline{1} 0\rangle+n \times 1 / 3\langle 01 \overline{1} 0\rangle$.

(3) $\{1 \overline{1} 00\} /\langle 11 \overline{2} 0\rangle$ tilt grain boundary

The $\langle 1 \overline{1} 00\rangle$ perfect edge dislocations dissociate into three $1 / 3\langle 1 \overline{1} 00\rangle$ partial dislocations with the $\{1 \overline{1} 00\}$ stacking faults of $I_{2}$ and $V$. The stacking fault energies were estimated to be $\mathrm{I}_{2}: 0.41-0.46 \mathrm{Jm}^{-2}$ and V: $0.33-0.37 \mathrm{Jm}^{-2}$.

(4) $(0001) /[1 \overline{1} 00]$ tilt grain boundary

The misorientation of the grain boundary is accommodated by the groups of the $1 / 3[0 \overline{1} 11]$, $1 / 3[10 \overline{1} 1]$, and $1 / 3[\overline{1} 101]$ perfect mixed dislocations. Each perfect dislocation dissociates into $1 / 18\langle\overline{4} 223\rangle$ and $1 / 18\langle\overline{2} 4 \overline{2} 3\rangle$ partial dislocations with the (0001) stacking fault. The stacking fault energy was estimated to be $0.58 \mathrm{Jm}^{-2}$.

(5) $\{\overline{1} 104\} /\langle 11 \overline{2} 0\rangle$ tilt grain boundary

The misorientation of the grain boundary is accommodated by $1 / 3\langle 1 \overline{2} 10\rangle$ perfect mixed dislocations and $1 / 3\langle\overline{1} 101\rangle$ perfect edge dislocations. The $1 / 3\langle 1 \overline{2} 10\rangle$ dislocations are not dissociated, whereas $1 / 3\langle\overline{1} 101\rangle$ edge dislocations dissociate into $1 / 18\langle\overline{4} 223\rangle$ and $1 / 18\langle\overline{2} 4 \overline{2} 3\rangle$ with the $(0001)$ stacking fault.

(6) $(0001) /[0001]$ twist grain boundary

The hexagonal network of the $1 / 3\langle 11 \overline{2} 0\rangle$ prefect screw dislocations is formed. The $1 / 3\langle 11 \overline{2} 0\rangle$ screw dislocation is not dissociated into partial dislocations.

Acknowledgments: The authors gratefully thank K. Peter D. Lagerlöf, Takahisa Yamamoto, Katsuyuki Matsunaga, Teruyasu Mizoguchi, Matthew F. Chisholm, Hitoshi Nishimura, and Yuki Kezuka for collaborative works on dislocations in alumina. A part of this study was supported by Grant-in-Aid for Specially promoted Research (Grant No. JP17H06094) from the Japan Society for the Promotion of Science (JSPS), the Elements Strategy Initiative 
for Structural Materials (ESISM) from the Ministry of Education, Culture, Sports, Science, and Technology in Japan (MEXT), Grant-in-Aid for Scientific Research on Innovative Areas “Nano Informatics" (Grant No. JP25106002 and JP25106003) from JSPS, “Nanotechnology Platform" (Project No. 12024046) of MEXT, and JSPS KAKENHI (Grant Nos. JP15H04145 and JP15K20959, and JP17K18983).

Author Contributions: Eita Tochigi drafted the manuscript. All the authors discussed and amended the manuscript.

Conflicts of Interest: The authors declare no conflict of interest.

\section{References}

1. Sutton, A.P.; Balluffi, R.W. Interfaces in Crystalline Materials; Clarendon Press: Oxford, UK, 1995.

2. Hirth, J.P.; Lothe, J. Theory of Dislocations, 2nd ed.; Krieger Publishing Company: Malabar, India, 1982.

3. Snow, J.D.; Heuer, A.H. Slip systems in $\mathrm{Al}_{2} \mathrm{O}_{3}$. J. Am. Ceram. Soc. 1973, 56, 153-157. [CrossRef]

4. Pletka, B.J.; Heuer, A.H.; Mitchell, T.E. Dislocation structures in sapphire deformed basal slip. J. Am. Ceram. Soc. 1974, 56, 136-139. [CrossRef]

5. Bilde-Sørensen, J.B.; Thölen, A.R.; Gooch, D.J.; Groves, G.W. Structure of $\langle 01 \overline{1} 0\rangle$ dislocation in sapphire. Philos. Mag. 1976, 33, 877-889. [CrossRef]

6. Mitchell, T.E.; Pletka, B.J.; Phillips, D.S.; Heuer, A.H. Climb dissociation in sapphire $\left(\alpha-\mathrm{Al}_{2} \mathrm{O}_{3}\right)$. Philos. Mag. 1976, 34, 441-451. [CrossRef]

7. Firestone, R.F.; Heuer, A.H. Creep deformation of $0^{\circ}$ sapphire. J. Am. Ceram. Soc. 1976, 59, 13-19. [CrossRef]

8. Lagerlöf, K.P.D.; Mitchell, T.E.; Heuer, A.H.; Rivière, J.P.; Cadoz, J.; Castaing, J.; Phillips, D.S. Stacking fault energy in sapphire $\left(\alpha-\mathrm{Al}_{2} \mathrm{O}_{3}\right)$. Acta Met. 1984, 32, 97-105. [CrossRef]

9. Lagerlöf, K.P.D.; Heuer, A.H.; Castaing, J.; Rivière, J.P.; Mitchell, T.E. Slip and twinning in sapphire. J. Am. Ceram. Soc. 1994, 77, 385-397.

10. Bilde-Sørensen, J.B.; Lawlor, B.F.; Geipel, T.; Pirouz, P.; Heuer, A.H.; Lagerlöf, K.P.D. On basal slip and basal twinning in sapphire $\left(\alpha-\mathrm{Al}_{2} \mathrm{O}_{3}\right)$ - I. Basal slip revisited. Acta Mater. 1996, 44, 2145-2152. [CrossRef]

11. Nakamura, A.; Yamamoto, T.; Ikuhara, Y. Direct observation of basal dislocation in sapphire by HRTEM. Acta Mater. 2002, 50, 101-108. [CrossRef]

12. Nakamura, A.; Matsunaga, K.; Tohma, J.; Yamamoto, T.; Ikuhara, Y. Conducting nanowires in insulating ceramics. Nat. Mater. 2003, 2, 453-456. [CrossRef] [PubMed]

13. Tochigi, E.; Kezuka, Y.; Nakamura, A.; Nakamura, A.; Shibata, N.; Ikuhara, Y. Direct observation of impurity segregation at dislocation cores in an ionic crystal. Nano Lett. 2017, 17, 2908-2912. [CrossRef] [PubMed]

14. Bouchet, D.; Lartigue-Korinek, S.; Molis, R.; Thibault, J. Yttrium segregation and intergranular defects in alumina. Philos. Mag. 2006, 86, 1401-1413. [CrossRef]

15. Lartigue-Korinek, S.; Bouchet, D.; Bleloch, A.; Colliex, C. HAADF study of relationship between intergranular defect structure and yttrium segregation in an alumina grain boundary. Acta Mater. 2011, 59, 3519-3527. [CrossRef]

16. Ikuhara, Y. Nanowire design by dislocation technology. Prog. Mater. Sci. 2009, 54, 770-791. [CrossRef]

17. Nakamura, A.; Mizoguchi, T.; Matsunaga, K.; Yamamoto, T.; Shibata, N.; Ikuhara, Y. Periodic Nanowire Array at the Crystal Interface. ACS Nano 2013, 7, 6297-6302. [CrossRef] [PubMed]

18. Matsunaga, K.; Nishimura, H.; Saito, T.; Yamamoto, T.; Ikuhara, Y. High-resolution transmission electron microscopy and computational analyses of atomic structures of [0001] symmetric tilt grain boundaries of $\mathrm{Al}_{2} \mathrm{O}_{3}$ with equivalent grain-boundary planes. Philos. Mag. 2003, 83, 4071-4082. [CrossRef]

19. Gemming, T.; Nufer, S.; Kurtz, W.; Rühle, M. Structure and chemistry of symmetrical tilt grain boundaries in $\alpha-\mathrm{Al}_{2} \mathrm{O}_{3}$ : I, Bicrystals with "clean" interface. J. Am. Ceram. Soc. 2003, 86, 581-589. [CrossRef]

20. Lartigue-Korinek, S.; Liagege, S.; Kisielowski, C.; Serra, A. Disconnection arrays in a rhombohedral twin in $\alpha$-alumina. Philos. Mag. 2008, 88, 1569-1579. [CrossRef]

21. Ikuhara, Y.; Nishimura, H.; Nakamura, A.; Matsunaga, K.; Yamamoto, T. Dislocation structures of low-angle and near- $\Sigma 3$ grain boundaries in alumina bicrystals. J. Am. Ceram. Soc. 2003, 86, 595-602. [CrossRef]

22. Nakamura, A.; Matsunaga, K.; Yamamoto, T.; Ikuhara, Y. Multiple dissociation of grain boundary dislocations in alumina ceramic. Philos. Mag. 2006, 86, 4657-4666. [CrossRef]

23. Shibata, N.; Chisholm, M.F.; Nakamura, A.; Pennycook, S.J.; Yamamoto, T.; Ikuhara, Y. Nonstoichiometric dislocation cores in $\alpha$-alumina. Science 2007, 316, 82-85. [CrossRef] [PubMed] 
24. Tochigi, E.; Shibata, N.; Nakamura, A.; Yamamoto, T.; Ikuhara, Y. TEM characterization of $2^{\circ}$ tilt grain boundary in alumina. Mater. Sci. Forum 2007, 561-565, 2427-2430. [CrossRef]

25. Tochigi, E.; Shibata, N.; Nakamura, A.; Yamamoto, T.; Lagerlöf, K.P.D.; Ikuhara, Y. Dislocation structure of $10^{\circ}$ low-angle tilt grain boundary in $\alpha-\mathrm{Al}_{2} \mathrm{O}_{3}$. Mater. Sci. Forum 2007, 558-559, 979-982. [CrossRef]

26. Tochigi, E.; Shibata, N.; Nakamura, A.; Yamamoto, T.; Ikuhara, Y. Partial dislocation configurations in a low-angle boundary in $\alpha-\mathrm{Al}_{2} \mathrm{O}_{3}$. Acta Mater. 2008, 56, 2015-2021. [CrossRef]

27. Tochigi, E.; Shibata, N.; Nakamura, A.; Mizoguchi, T.; Yamamoto, T.; Ikuhara, Y. Structures of dissociated $\langle 1 \overline{1} 00\rangle$ dislocations and $\{1 \overline{1} 00\}$ stacking faults of alumina $\left(\alpha-\mathrm{Al}_{2} \mathrm{O}_{3}\right)$. Acta Mater. 2010, 58, 208-215. [CrossRef]

28. Tochigi, E.; Shibata, N.; Nakamura, A.; Yamamoto, T.; Ikuhara, Y. Dislocation structures in a $\{\overline{1} 104\} /\langle 11 \overline{2} 0\rangle$ low-angle tilt grain boundary of alumina $\left(\alpha-\mathrm{Al}_{2} \mathrm{O}_{3}\right)$. J. Mater. Sci. 2011, 46, 4428-4433. [CrossRef]

29. Tochigi, E.; Kezuka, Y.; Shibata, N.; Nakamura, A.; Ikuhara, Y. Structure of screw dislocations in a $(0001) /[0001]$ low-angle twist grain boundary of alumina $\left(\alpha-\mathrm{Al}_{2} \mathrm{O}_{3}\right)$. Acta Mater. 2012, 60, 1293-1299. [CrossRef]

30. Tochigi, E.; Nakamura, A.; Mizoguchi, T.; Shibata, N.; Ikuhara, Y. Dissociation of the $1 / 3\langle\overline{1} 101\rangle$ dislocation and formation of the anion stacking fault on the basal plane in $\alpha-\mathrm{Al}_{2} \mathrm{O}_{3}$. Acta Mater. 2015, 91, $152-161$. [CrossRef]

31. Tochigi, E.; Findlay, S.D.; Okunishi, E.; Mizoguchi, T.; Nakamura, A.; Shibata, N.; Ikuhara, Y. Atomic structure characterization of stacking faults on the $\{1 \overline{1} 00\}$ plane in $\alpha$-alumina by scanning transmission electron microscopy. AIP Conf. Proc. 2016, 1763, 050003.

32. Tochigi, E.; Mizoguchi, T.; Okunishi, E.; Nakamura, A.; Shibata, N.; Ikuhara, Y. Dissociation reaction of the $1 / 3\langle\overline{1} 101\rangle$ edge dislocation in $\alpha-\mathrm{Al}_{2} \mathrm{O}_{3}$. J. Mater. Sci. 2018, in press. [CrossRef]

33. Nakamura, A.; Tochigi, E.; Shibata, N.; Yamamoto, T.; Ikuhara, Y. Structure and configuration of boundary dislocations on low angle tilt grain boundaries in alumina. Mater. Trans. 2009, 50, 1008-1014. [CrossRef]

34. Chung, D.H.; Simmons, G. Pressure and temperature dependences of isotropic elastic moduli of polycrystalline alumina. J. Appl. Phys. 1968, 39, 5316-5326. [CrossRef]

35. Gieske, J.H.; Barsch, G.R. Pressure dependence of elastic constants of single crystalline aluminum oxide. Phys. Stat. Sol. 1968, 29, 121-131. [CrossRef]

36. Kenway, P.R. Calculated stacking-fault energies in $\alpha-\mathrm{Al}_{2} \mathrm{O}_{3}$. Philos. Mag. B 1993, 68, 171-183. [CrossRef]

37. Jhon, M.H.; Glaeser, A.M.; Chrzan, D.C. Computational study of stacking faults in sapphire using total energy methods. Phys. Rev. B 2005, 71, 214101. [CrossRef]

38. Okunishi, E.; Ishikawa, I.; Sawada, H.; Hosokawa, F.; Hori, M.; Kondo, Y. Visualization of light elements at ultrahigh resolution by STEM annular bright field microscopy. Microsc. Microanal. 2009, 15, 164-165. [CrossRef]

39. Findlay, S.D.; Shibata, N.; Sawada, H.; Okunishi, E.; Kondo, Y.; Yamamoto, T.; Ikuhara, Y. Robust atomic resolution imaging of light elements using scanning transmission electron microscopy. Appl. Phys. Lett. 2009, 95, 191913. [CrossRef]

40. Marinopoulos, A.G.; Elsässer, C. Density-functional and shell-model calculations of the energies of basal-plane stacking faults in sapphire. Philos. Mag. Lett. 2001, 81, 329-338. [CrossRef]

41. Duesberya, M.S.; Vitek, V. Plastic anisotropy in b.c.c. transition metals. Acta Mater. 1998, 46, 1481-1492. [CrossRef]

(C) 2018 by the authors. Licensee MDPI, Basel, Switzerland. This article is an open access article distributed under the terms and conditions of the Creative Commons Attribution (CC BY) license (http://creativecommons.org/licenses/by/4.0/). 